\title{
Klima-Änderungen im Pleistozän: Isotopen- untersuchungen an fossilen Seesedimenten aus dem Holstein-Interglazial Ost-Polens
}

\author{
Jerzy Nitychoruk, Jochen Hoefs \& JÜrgen SChNEIDER*) \\ - Holsteinian interglacial, Climate change, lacustrine sediments, C- and O-isotopes -
}

\begin{abstract}
Kurzfassung: In Ost-Polen erbohrte Seesedimente mit einer Mächtigkeit bis zu $55 \mathrm{~m}$ (Ossówka-See) dokumentieren das ganze Holstein-Interglazial und die Anfangsperiode der Saale-Eiszeit. An ausgewählten Bohrkernen der Seen von Ossówka und Wilczyn wurden palaeobiologische (Malakofauna, Palynologie und Pflanzen-Makroreste) und Isotopen-Untersuchungen durchgeführt. Die für den längsten $(55 \mathrm{~m})$ und vollständigsten Bohrkern nahe der Ortschaft Ossówka bestimmten C- und O-Isotopenwerte betragen:

213C: Minimalwerte bis $-6,4 \%$ für Ablagerungen am Beginn des Interglazials, Maximalwerte bis $+10,0 \%$ für Ablagerungen aus der kalten Frühglazialperiode;

218O: Maximalwerte bis $-3,6 \%$ für Ablagerungen aus dem ersten Abschnitt des Interglazial-Optimums, Minimalwerte bis -10,1\% für Ablagerungen aus der kältesten Periode unmittelbar vor der nächsten Vereisung. Generell gibt der Kurvenverlauf der O-Isotopenwerte gut die palynologisch dokumentierten Klimaveränderungen wieder. Im Profil sind jedoch zwei Perioden zu beobachten, in denen das Isotopenbild nicht mit der palynologischen Aussage übereinstimmt, einmal im klimatischen Interglazial-Optimum und zum anderen im jüngeren Teil des frühen Saale-Glazials
\end{abstract}

1. Während des klimatischen Optimums des Holsteininterglazials (Pollen-Zone $\mathrm{G}$ und $\mathrm{H}$ ) sprechen die Isotopenkurven der Seesedimente für relativ kühle Klimaverhältnisse. Dies kann durch eine Zunahme der Niederschlagsmenge, die zu einer Seespiegel-Erhöhung führte und/oder durch den Einfluß von isotopisch leichten Zuflüssen erklärt werden.

2. Im oberen Teil des Profils, der eine kühle, der Vereisung vorangehende Phase darstellt, erreichen die $213 \mathrm{C}$ und $218 \mathrm{O}-$-Isotope unerwartet hohe Werte, was möglicherweise auf die Redeposition von "warmen" interglazialen Ablagerungen und/oder auf eine Zunahme der Evaporation unter trockenen Steppenklima-Bedingungen mit Seespiegel-Tiefständen zurückzuführen ist. Ab-

\footnotetext{
*) Anschriften der Verfasser: Dr. J. Nitychoruk, Institute of Geology, Warsaw University, Al. Øwirki i Wigury, 02-089 Warsaw, Poland, Alexander-von-Humboldt-Stipendiat, Prof. Dr. J. Hoefs, Institut für Geochemie, Universität Göttingen, Goldschmidtstraße 1, D-37077 Göttingen, Germany, Prof. Dr. J. SchneIDer, Institut für Geologie und Dynamik der Lithosphäre, Universität Göttingen, Goldschmidtstraße 3, D-37077 Göttingen, Germany
}

kühlungsphasen fallen mit der Verschiebung der Sauerstoffisotopenverhältnisse in Richtung einer ${ }^{18 \mathrm{O}-\text { Ver- }}$ armung zusammen.

\section{[Climate change during Pleistocene: Isotopic investigations of fossil lacustrine sediments of the Holsteinian interglacial of Eastern Poland]}

Abstract: Lacustrine sediments of the Holsteinian interglacial and the early part of the Saalian glaciation from Eastern Poland are exceptional in Europe:

- they have a maximum thickness of at least $55 \mathrm{~m}$, - they are very homogeneous (lake marl and gyttja), - they have been geologically and palaeobiologically investigated in great detail (pollen analysis, macrofossils, mollusc fauna).

Oxygen and carbon isotope composition of these sediments allow a precise reconstruction of climatic variations to be made: Minimum values of $\partial{ }^{13} \mathrm{C}(-6.4 \%)$ were found in the earliest part of the interglacial period, maximum $(+10.0 \%)$ values could be detected at the onset of the Saalian glaciation. The distribution of the $\partial 18 \mathrm{O}$ values follows an inverse trend. The maximum values $(-3.6 \%)$ occur at the initial period of the interglacial and the minimal $(-10.1 \% 0)$ are associated with a progressive temperature decrease at the end of the interglacial.

Generally the isotope data satisfactorily reflect the palynologically well-documented climatic changes. However there are two sections in the profile, where this correlation is poor:

1. At the palynologically defined interglacial climatic optimum the 180 curves indicate relatively cool climatic conditions.

2. In the upper part of the sediment profile, which probably reflects the onset of the Saalian glaciation, the $18 \mathrm{O}$ and $13 \mathrm{C}$ - isotope curves have relatively high values, indicating too high temperatures.

For the first case an inflow of $18 \mathrm{O}$ depleted groundwater or/and an increase of precipitation with a rise of the lake level is postulated.

In the second case the poor correlation is quite likely caused by a redeposition of sediments of the warm interglacial period, and/or by an enrichment of $18 \mathrm{O}$ and ${ }^{13} \mathrm{C}$ due to intense evaporation. 


\section{Einleitung}

Klimafluktuationen im Pleistozän, von warmen Interglazialen bis zu Eiszeiten, spiegeln sich in Änderungen der Sauerstoffisotopenverhältnisse $(18 \mathrm{O} / 16 \mathrm{O})$ in Karbonaten wider, die durch temperaturabhängige Fraktionierungsprozesse zwischen Karbonat und Wasser verursacht werden. Dies belegen zahlreiche Untersuchungen an marinen Sedimentprofilen (z. B. SHACKLETON \& Opdyke 1973, Imbrie et al. 1973, Pisias et al. 1984, MARTINSON at al. 1987, SHACKLETON et al. 1992), von denen in der Regel lückenlose Profile existieren. Isotopen-Untersuchungen kontinentaler Seesedimente bringen nicht immer zufriedenstellende Ergebnisse, vor allem wegen der wechselnden Sedimentationsbedingungen und einer größeren Variabilität der Wasserzusammensetzung, die auf die geringe Größße der meisten kontinentalen Seen zurückzuführen ist. Andere Faktoren sind die Evaporationsverhältnisse, das Vorhandensein von Wasserzuflüssen, die Wassertiefe sowie die Entfernung vom Ozean (Eicher \& Siegenthaler 1976, 1981, EICHER et al. 1991, ROZANSKI et al. 1993). Klimatische und hydrologische Bedingungen sind die wichtigsten Faktoren, welche die Isotopen-Verhältnisse der Seekarbonate beeinflussen. Die oft vorkommenden Sedimentationslücken, bzw. lokale hydrogeologische Instabilitäten erschweren die Interpretation der IsotopenKurven aus Seen oder machen sie sogar unmöglich (Beaulieu et al. 1994).

$\mathrm{Zu}$ besseren Ergebnissen führen die Untersuchungen an Tropfsteinen aus Höhlen z. B. in Nevada (WinOGRaD et al. 1992) sowie in Nord-England (GAsCoyne 1992). Obwohl sich das durch Tropfsteinuntersuchungen gewonnene Isotopenbild von dem unterscheidet, welches die Untersuchungen der marinen Profile erbracht haben,

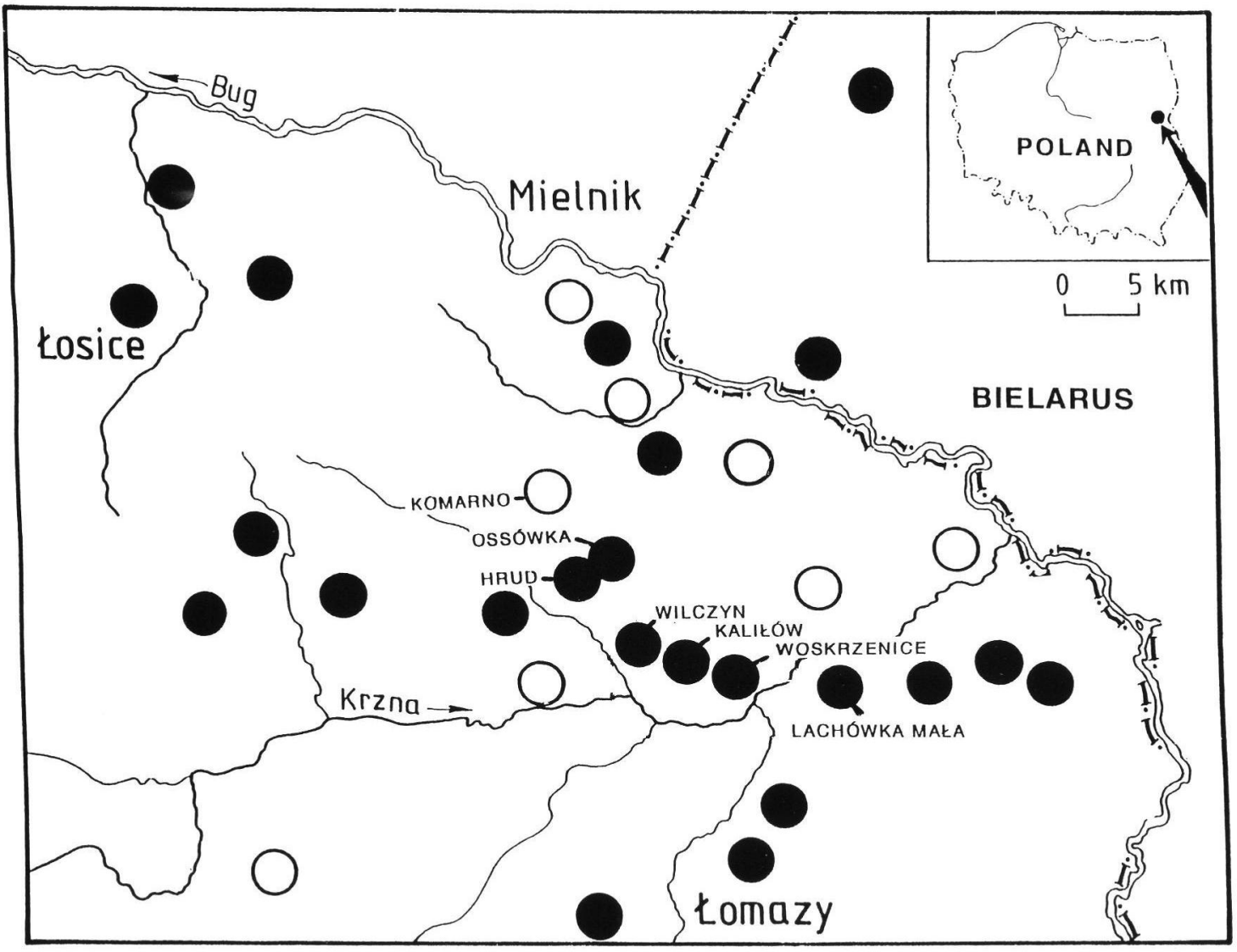

Abb. 1: Lokalitäten mit fossilen Seesedimenten aus dem Holstein-Interglazial in Ost-Polen (nach Nitychoruk 1994, AlBRycht et al. 1997). Mit schwarzen Punkten sind die Lokalitäten mit Karbonatablagerungen gekennzeichnet.

Fig. 1: Sites with fossil lake sediments dating from to the Holsteinian Interglacial in eastern Poland (after NityCHORUK 1994, AlBRyChT et al. 1997). Black circles indicate locations with carbonate sediments. 
können die O-Isotopen-Kurven aus den Höhlen und Ozeanen mit Hilfe der Th/U-Datierungen verglichen werden.

Das bisher genaueste Isotopen-Bild der paläoklimatischen Veränderungen wurde für die letzten 140 ka des Pleistozäns, darunter für die WeichselEiszeit und das Eem-Interglazial an grönlandischen Eisbohrkernen und an Seesedimenten Mitteldeutschlands gewonnen (DANSGAARD et al. 1993, Johnsen et al. 1995, LitT et al. 1996). Viel schwieriger ist es aber, ältere, palynologisch gut datierte Interglaziale dem entsprechenden Isotopen-Stadium zuzuordnen, weil sie keine charakteristischen Isotopen-Kurven zeigen. Deshalb wird z. B. das Holstein-Interglazial, das aus den Untersuchungen der fossilen Sedimente der kontinentalen Seen gut bekannt ist, dem 11. (SARNTHein et al. 1986, BeAulieu \& ReIlle 1995), 9. (ZAGWIJN 1992) oder 7. (LINKE et al. 1985) O-IsotopenStadium aus Tiefseeprofilen zugeordnet.

\section{Bisherige Untersuchungen an ostpolnischen Seen}

Ende der 80er und Anfang der 90er Jahre wurde in Ost-Polen eine fossile Seenplatte (NiTychORuK 1994, AlBrycht et al. 1997, Nitychoruk et al. 1997) entdeckt und beschrieben (Abb. 1). Die Seen sind in Form von Rinnensee-Ketten von einigen bis zu mehreren Kilometern Länge aufgereiht (Abb. 1) und erstrecken sich in SW-NE bzw. in W-E Richtung, parallel zu den paläozoischen Strukturen (Nitychoruk 1994). Diese Seenkette liegt in einem Bereich zwischen wechselnd kontinental bzw. Ozeanisch beeinflußtem Klima. Somit sind nicht nur Klima-Wechsel in der Zeit, sondern auch im Raum zu berücksichtigen. Sowohl die Ergebnisse der palynologischen Analyse (LINDNER et al. 1991, KRupinski \& NitychoruK 1991, NityCHORUK 1994, KRUPINSKI 1995, BinKA \& NiTYCHORUK 1995, 1996, NiTYCHORUK \& BiNKA 1994, NiTYCHORUK et al. 1997) als auch die Bestimmungen der in Seesedimenten gefundenen Schnecken - Viviparus diluviamus (KunTH.) und Lithoglyphus pyramidatus Mildf. (LinDNer et al. 1991) -, deren Aussterben mit dem Ausklang des Holstein-Interglazials zusammenfällt, ermöglichen es, diese Seenplatte in das Holstein-Interglazial zu stellen. In den bis heute ungefähr 30 beschriebenen Lokalitäten (Abb. 1) bestehen die fossilen Seesedimente aus Gyttja und Seekreide mit 30 m Mächtigkeit oder aus $\mathrm{C}_{\text {org }}$-reichen Tonen mit eingeschalteten verschiedenen Torfarten mit einer Mächtigkeit von einigen Metern (NITYCHORUK 1994). Aus den palynologischen Untersuchungen kann geschlossen werden, daß die ganze Periode des Holstein-Interglazials und das danach folgende Frühglazial in den kalkigen Seesedimenten enthalten sind (Abb. 2, s. BinKA \& NitYCHORUK 1996).

Die fossilen Karbonatablagerungen sind in den obersten 2-3 $\mathrm{m}$ anzutreffen (Abb. 3). Die fossilen Seesedimente aus dem Holstein-Interglazial liegen auf dem Geschiebelehm und den glazifluvialen Sanden der Elster-Eiszeit (Abb. 3). Der Ursprung dieser Seen kann im Schmelzen des Inlandeises vermutet werden, was die an der Basis der Karbonatserien erhaltenen Silte und Tone bestätigen. Die bislang für zwei Seen durchgeführten Datierungen dieser Tone (mit Hilfe der Thermolumineszenz-Methode) haben unterschiedliche Alter zwischen 430 (? zu hoch) und $230 \mathrm{ka}$ (KRUPINSKI 1995) ergeben und bestätigen damit diesen zeitlichen Rahmen. Glazialablagerungen, die über den Seeablagerungen liegen, entstanden durch den Eiszerfall der Gletscher, die nach dem Holstein-Interglazial das Gebiet erreichten. Sie haben ein Alter zwischen $163 \pm 24$ ka und $186 \pm$ $28 \mathrm{ka}$ (Abb. 3) und datieren diese Sedimente auf die Saale-Eiszeit (Nitychoruk 1994).

1996 wurde eine neue Serie von Bohrungen mit vollständigen Kernen durchgeführt. Eine dieser Bohrungen (OS. 1/96) ergab in dem Ort Ossówka eine vollständige Sediment-Abfolge mit einer Mächtigkeit von $55 \mathrm{~m}$ (Abb. 2, 3). Der gewonnene Kern enthält in seinem unteren Teil einen $17 \mathrm{~m}$ langen Abschnitt mit einer Wechsellagerung (dunkel-hell). Die detaillierten palynologischen Bestimmungen ergaben, daß der $17 \mathrm{~m}$ lange laminierte Kernteil (35-52 m - Abb. 2) während des Holstein-Interglazials entstand. Im frühglazialen (nicht laminierten) Teil des Kerns herrschten boreale Kiefer- und Birken-Wälder und subarktische Verhältnisse vor. Das in dem $55 \mathrm{~m}$ langen Kern (OS. 1/96 - Abb. 2) enthaltene palynologische Bild ist von allen Bohrungen, die bisher für das Holstein-Interglazial in diesem Teil Europas gewonnen wurden, am vollständigsten und ist vergleichbar mit früheren Untersuchungen (KRupinsKI 1995) an Kernen von 29 m (OS. 2/90) und $34 \mathrm{~m}$ (OS. 1/90) Länge (Abb. 2, 3)

\section{Analyse-Methoden}

An diesen besonders mächtigen und vollständig geologisch, palynologisch sowie malakologisch bearbeiteten fossilen Seesedimenten wurde die 


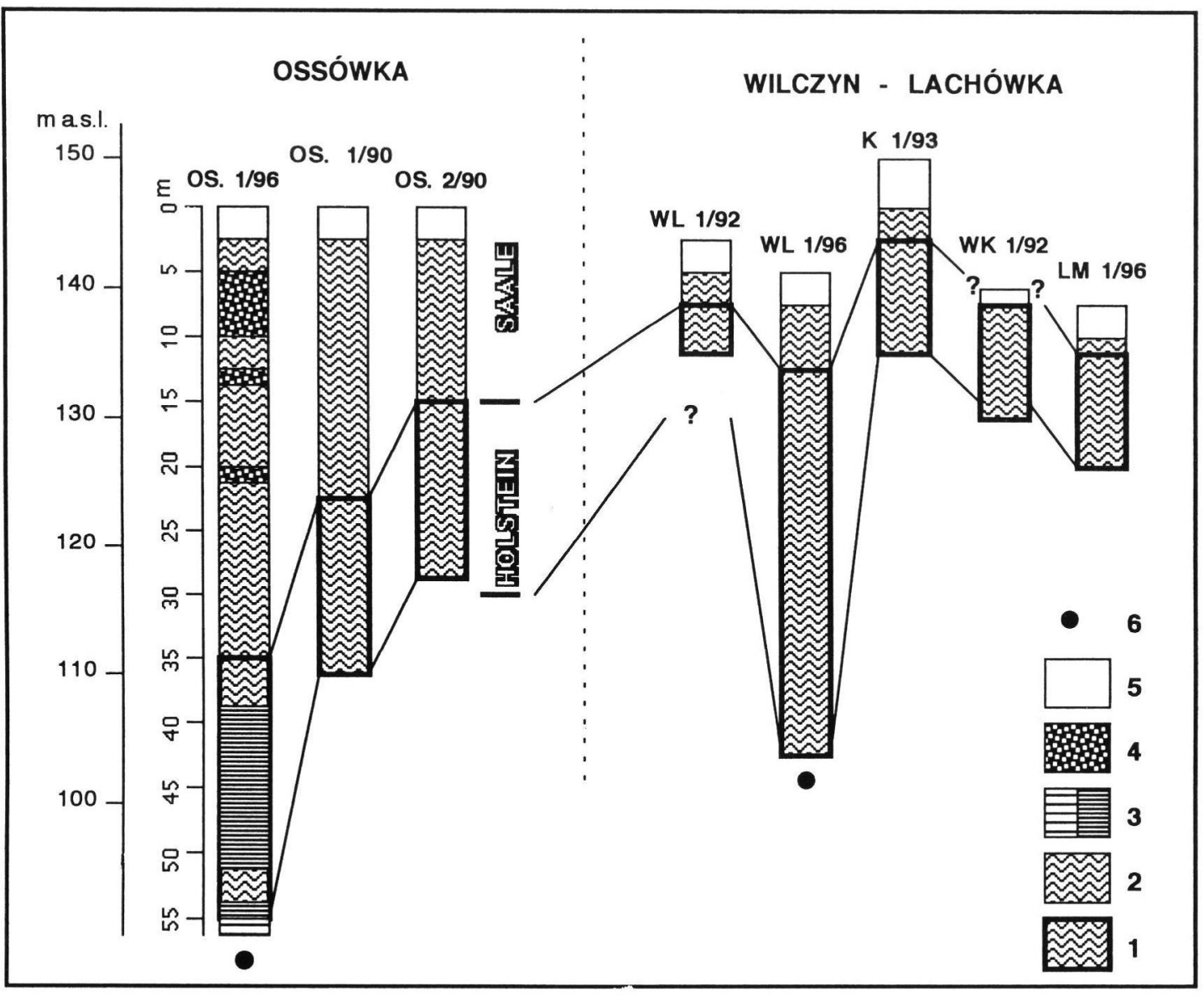

Abb. 2: Ausgewählte palynologisch bearbeitete Kern-Profile. 1 - Karbonat-Ablagerungen aus dem Holstein-Interglazial; 2 - Karbonat-Ablagerungen, Gyttja und Seekreide aus der frühen Saale-Zeit; 3 - Laminierte Sedimente; 4 - Malakofauna; 5 - Deckschicht; 6 - Kernprofile mit Analysen der Isotope 13C und $18 \mathrm{O}$ (die zeitliche Zuordnung basiert auf Pollenuntersuchungen).

Fig. 2: Selected palynologically analysed core-profiles. 1 - carbonate sediments of the Holsteinian Interglacial; 2 - carbonate sediments, gyttya and lacustrine chalk of the early Saalian Glaciation; 3 - laminated sediments; 4 - mollusc shells; 5 - non lacustrine cover sediments; 6 - core profiles with oxygen and carbon isotope analyses (correlation based on palynological analyses).

Isotopenzusammensetzung (18O, 13C) bestimmt. Dazu wurden zwei Kerne aus den fossilen Seen Ossówka (OS. 1/96, 55 m Länge) und Wilczyn (WL 1/96, 35 m Länge) ausgewählt (Abb. 2, 3). Die Kerne wurden systematisch im Abstand von einem halben Meter beprobt und auf ihre Isotopenzusammensetzung untersucht. Für jeden Kern lagen bereits bei Beginn der Untersuchung umfangreiche palynologische Daten vor, die eine Korrelation der Isotopenwerte mit den palynologischen Daten ermöglichen.

Die C- und O-Isotopenzusammensetzung wurde nach der klassischen Methode von McCrEA (1950) bestimmt. Das $\mathrm{CO}_{2}$ wurde mit Hilfe eines Gasmassenspektrometers Finnigan-MAT 251 im Geochemischen Institut der Universität Göttingen analysiert.

Die 13C- und 180-Konzentrationen der untersuchten Proben werden wie üblich als $\partial$-Werte ausgedrückt und sind in \%o auf den Standard PDB bezogen. Der analytische Fehler für die $\partial^{13} \mathrm{C}$-Werte beträgt etwa $\pm 0,1 \%$, bzw. für die $\partial 18 \mathrm{O}$-Werte $\pm 0,2 \%$.

Aufgrund der größeren Vollständigkeit wird im folgenden insbesondere das Profil OS. 1/96 (Abb. 2, 3) diskutiert. 


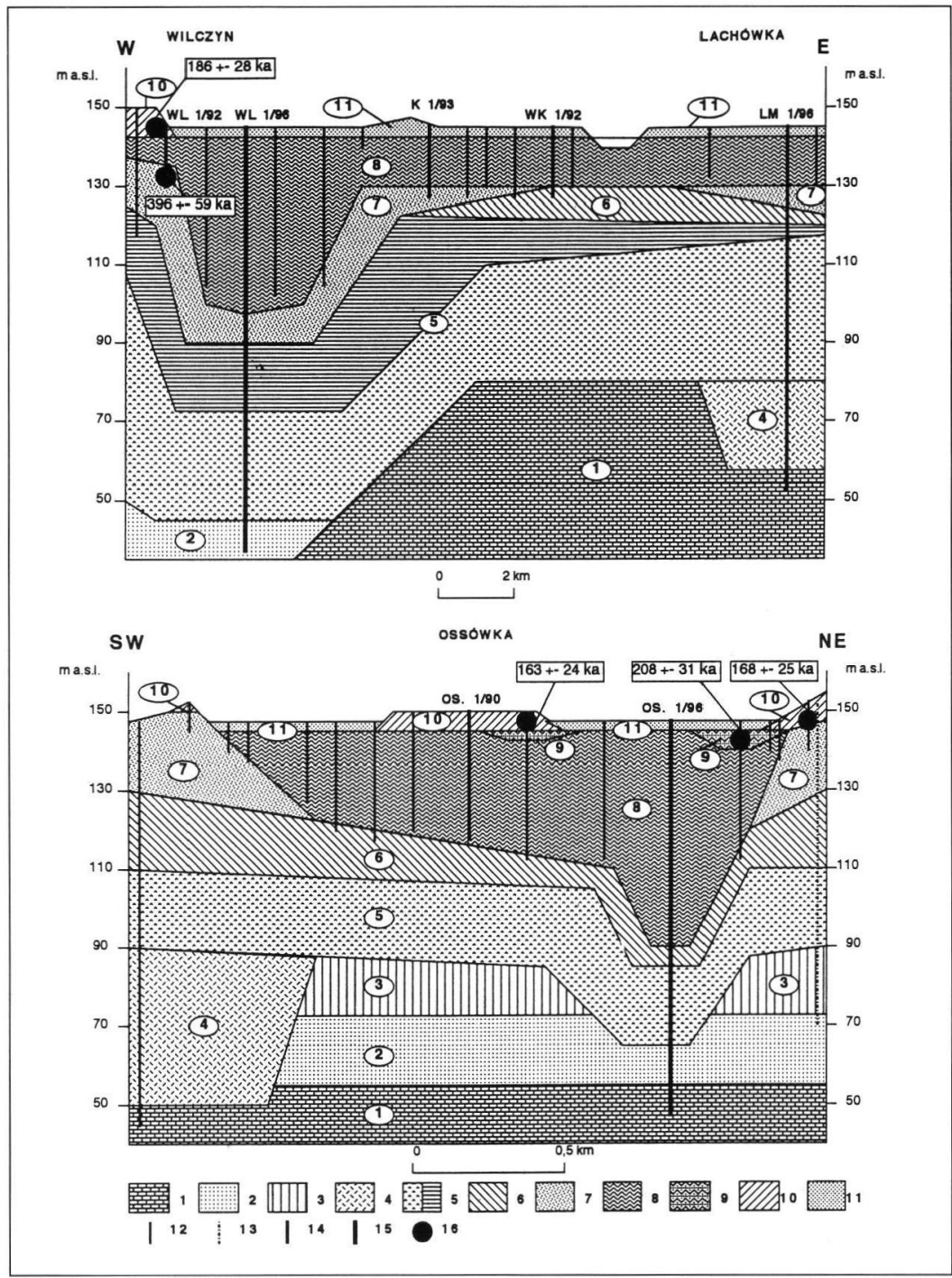

Abb. 3: Geologisches Profil durch die Lokalitäten Wilczyn-Lachówka und Ossówka. KREIDE: 1 - Mergel; TERTIÄR: 2 - Glaukonitsand; QUARTÄR: Menap-Vereisung: 3 - Geschiebelehm; Cromer-Interglazial: 4 - Sand und Silt mit Malakofauna und organischen Resten; Elster-Vereisung: 5 - Sand Silt und Ton mit organischen Resten, 6 - Geschiebelehm, 7 - grobkörniger Sand; Holstein-Interglazial: 8 - Gyttja und Seekreide; Saale-Glazial: 9 - Silt, 10 - Geschiebelehm; Saale-Glazial bis Holozän: 11 - Sand und Silt, 12 - Bohrungen; 13 - Bohrungen aus dem Profil; 14 - palynologisch bearbeitete Kernprofile; 15 - Kernprofile mit Analysen der Isotope 13C und 18O; 16 - TL -Datierungen (nach NiTYCHORUK 1994, BinKA et al. 1997).

Fig. 3: Geological section of the Wilczyn-Lachówka and Ossówka sites. CRETACEOUS: 1 - marl; TERTIARY: 2 - glauconite sand; QUATERNARY: Menap Glaciation: 3 - till; Cromer Interglacial: 4 - sand and silt with mollusc shells and organic detritus; Elster Glaciation: 5 - sand, silt and clay with organic detritus, 6 till; 7 - coarse-grained sand; Holstein Interglacial: 8 - gyttja and lacustrine chalk; Saale Glaciation: 9 - silt, 10 - till; Saale Glaciation - Holocene: 11 - sand and silt, 12 - coring site, 13 - coring site outside the profile, 14 - core profiles with palynological analyses, 15 - core profile with oxygen and carbon isotope analyses, 16 - TL age (after Nitychoruk 1994, Binka et al. 1997)

\section{Palynologische Befunde}

Zum besseren Verständnis der Isotopendaten soll zunächst das palynologische Bild der Vegetations-Änderungen im Holstein-Interglazial und in der Anfangsphase der folgenden Kaltzeit zusammengefaßt werden (s. BINKA \& NITYCHORUK 1995, 1996, KRUPINSKI 1995).

In der Anfangsphase des Holstein-Interglazials, nach einem noch kühlen Klima der Endphase der Eiszeit, herrschte ein boreales Klima. Vertreten wird es durch die PollenZonen (Abb. 4, 5): I (Zone A - Betula- NAP - Birke-NAP, Zone B - BetulaPinus - Birke-Kiefer) und II (Zone C - und Zone D Picea-Alnus - Fichte-Erle). In der Pollenperiode I erwärmte sich das Klima allmählich, aber deutlich, es besaß jedoch noch kontinentale Merkmale. Die Kontinentalität des Klimas zeigt sich durch einen trockenen und warmen Sommer, einen frostigen und langen Winter, eine kurze Vegetationsperiode sowie eine lange Zeit, in der die Seen mit einer Eisschicht bedeckt waren.

In der Pollenperiode II herrschte ein ziemlich mildes und feuchtes Klima mit warmen Sommern und langen, feuchten und ziemlich kalten Wintern. Im klimatischen Optimum des Interglazials (Pollen-Periode III) wuchsen Mischwälder und es herrschte ein gemäßigtes Klima. In den 


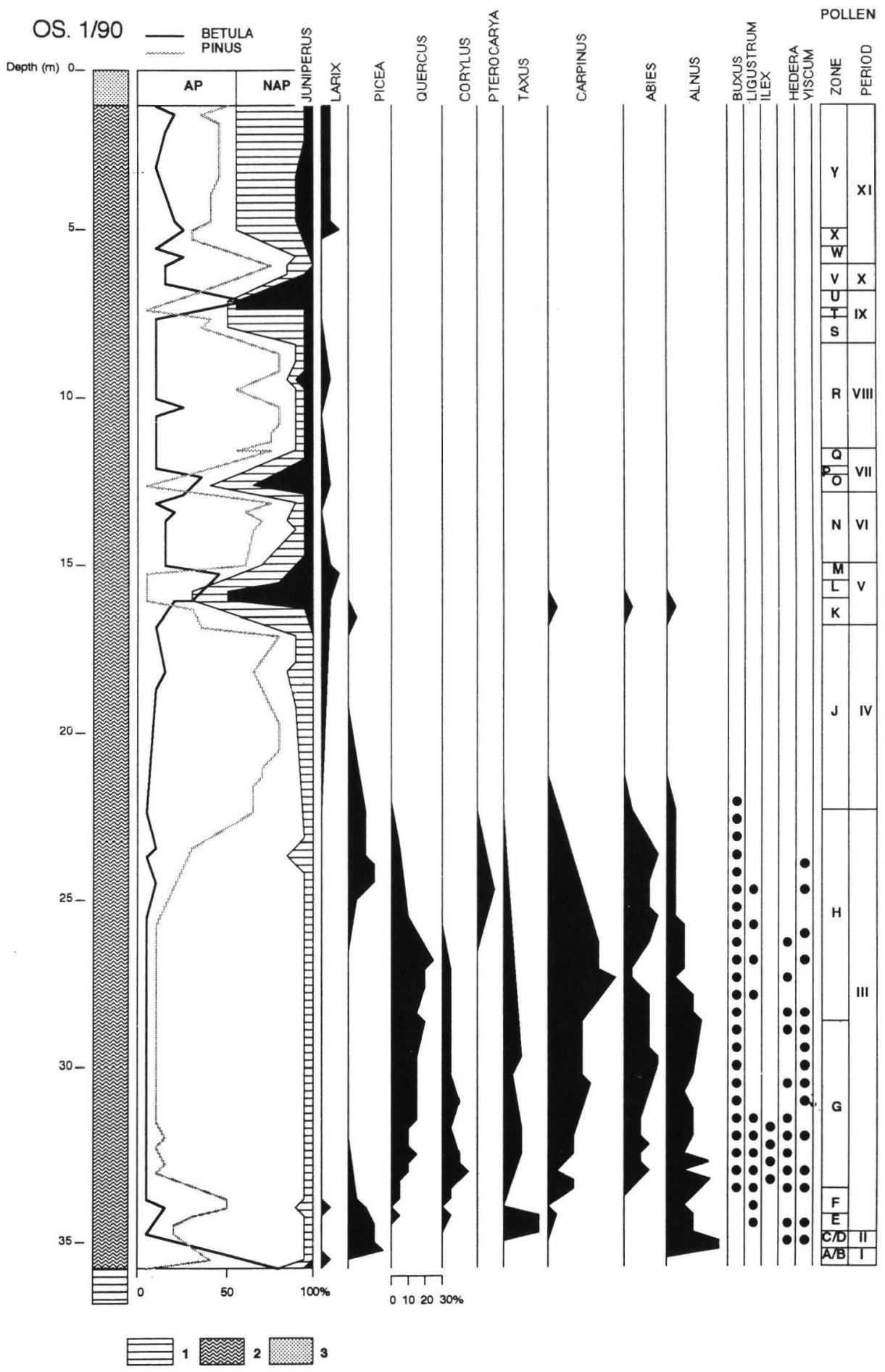

Abb. 4: Pollendiagramm des Holstein-Interglazials für Lokalität Ossówka 1/90 - s. Abb. 2. (vereinfacht nach KRUPINSKI 1995).

1 - Ton, 2 - Kalkgyttja und Seekreide, 3 - Torf und Sand

Fig. 4: Pollen diagram of the Holsteinian Interglacial site of Ossówka site 1/90 (simplified, based on KruPINSKI 1995). 

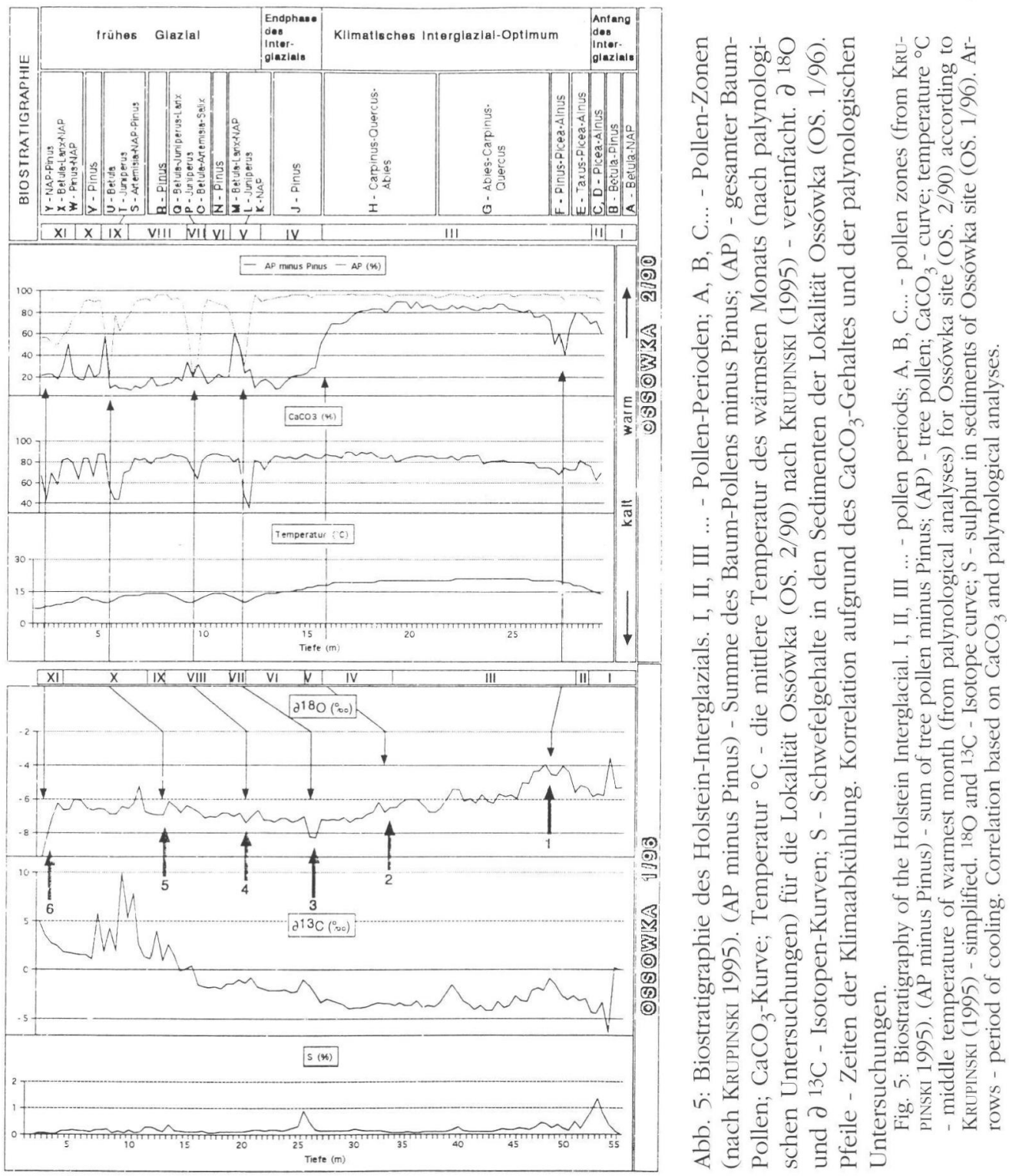

einzelnen Pollenzonen dominierten bestimmte Bäume und es herrschten folgende klimatische Verhältnisse:

Zone E - Taxus-Picea-Alnus (Eibe-Fichte-Erle) typische Vertreter für ein Klima mit ausgeprägten ozeanischen Merkmalen, die durch steigende Feuchtigkeit und zunehmende Niederschläge, durch einen ziemlich langen und feuchten Sommer und milden Winter sowie durch eine verlängerte Vegetationsperiode charakterisiert sind. Für das milde Klima spricht auch das Vorkommen von Hedera und Viscum.

Zone F - Pinus-Picea-Alnus (Kiefer-Fichte-Erle) kontinentales Klima ohne Hedera und Viscum, mit geringeren Niederschlägen und verringerter
Feuchtigkeit und mit tieferen Temperaturen. Zone G - Abies-Carpinus-Quercus (Tanne-Hainbuche-Eiche) - erneute Ozeanität des Klimas - milde und kurze Winter, lange Vegetationsperiode mit reichlichen Niederschlägen. Wichtige Klimaindikatoren sind Ilex, Hedera und Viscum. Zone H Carpinus-Quercus-Abies (Weißbuche-Eiche-Tanne). Das Klima in dieser Pollenzone war nicht einheitlich. In ihrem älteren Abschnitt besaß das Klima ozeanische Merkmale - hohe Feuchtigkeit und eine leicht niedrigere Sommertemperatur im Vergleich zu der vorangehenden Periode. In dem jüngeren Abschnitt dagegen traten kontinentale Merkmale mit verlängerten und kühleren Wintern auf. Indikatoren wintermilder Klimate fehlen. 
Die Endphase des Interglazials wird durch die Pollen-Periode IV vertreten, für die boreale Kiefernwälder charakteristisch sind (Abb. 4).

$\mathrm{Zu}$ dieser Zeit war das Klima noch feucht und ziemlich mild. Allmählich wechselte es zu einem immer trockener und kälter werdenden Klima mit langen frostigen Winterzeiten und trockenen, nicht besonders warmen Sommern. Für diese Deutung spricht die Entwicklung der Steppenvegetation. Im frühen Glazial wechselte das Klima zu subarktischen Verhältnissen (Pollen-Periode V), in der Sträucher, Gräser u.a. dominierten. In der Pollenzone K - NAP (s. Abb. 5) herrschte ein trockenes kaltes kontinentales Klima mit einer langen Winterperiode sowie mit einer trockenen und kurzen Vegetationsperiode.

Zone L - Juniperus (Wacholder) - zeigt eine geringe Klimaverbesserung, die sich in einer steigenden Feuchtigkeit äußerte, sowie in der Reduktion der Temperaturamplitude zwischen dem wärmsten und dem kältesten Monat im Jahr und in der Verlängerung der Vegetationsperiode. Gegen Ende der Periode $\mathbf{V}$ erscheinen Wälder (Zone M - Betula-Larix - Birke-Lärche). Das Klima verbessert sich deutlich, es wird feuchter und wärmer mit einer verlängerten Vegetationsperiode.

Die Zonen L und M sind eine typische Einwanderungsfolge von der Kaltzeit zur Warmzeit. Eine kurze Entwicklungsphase der Kiefernwälder in der Periode VI (Zone N - Pinus - Kiefer), als es zu einer Rückkehr zum borealen Klima mit kontinentalen Merkmalen kam, wird durch eine erneute Abkühlung (Periode VII) unterbrochen.

Die Abkühlung verursachte eine Expansion von Sträuchern und Kräutern in einer entsprechenden Einwanderungsfolge (Zone O - Betula-ArtemisiaSalix - Birke-Beifuß-Weide, Zone P - Juniperus Wacholder, Zone Q - Betula-Juniperus-Larix-Pteridium - Birke-Wacholder-Lärche-Pteridium). Die Pollen-Perioden VIII (Zone R - Pinus) und X (Zone $\mathrm{V}$ - Pinus) stellen den Kiefernwald als den dominierenden Bestandteil der Flora dar. Eine Abkühlungsphase trennt diese Perioden voneinander: IX (Zone S - Adlerfarn-NAP-Pinus, Zone T - Juniperus, Zone U - Betula) und XI (Zone W Pinus-NAP, Zone $\mathbf{X}$ - Betula-Larix- NAP, Zone Y-NAP-Pinus). Diese Abkühlungsphase bildet den nächsten Zeitabschnitt, in dem ein subarktisches Klima mit stark kontinentalen Merkmalen herrschte und der das bevorstehende nächste Inlandeis ankündigte.

\section{Interpretation der ${ }^{13} \mathrm{C}$ - und $18 \mathrm{O}$-Isotopen- Kurven und Vergleich mit den palynologischen Untersuchungen}

Wie aus den palynologischen Untersuchungen geschlossen werden kann, überdecken die gemessenen Isotopenkurven das ganze Interglazial in Ossówka, samt dem einige zehntausend Jahre dauernden frühglazialen Zeitabschnitt. In der Lokalität Wilczyn entsprechen die Isotopenkurven dagegen nur dem interglazialen Zeitabschnitt (Abb. 6). Beide Kurven zeigen einen ähnlichen Verlauf trotz gewisser Unterschiede in den absoluten Werten. Die beobachtete Korrelation der $13 \mathrm{C}$ - und $18 \mathrm{O}-$ Werte in Ossówka ist typisch für Seekarbonate in hydrologisch geschlossenen Gewässern. Dagegen ist eine solche Korrelation in Wilczyn wesentlich schwächer ausgeprägt und spricht eher für ein offenes System (EICHER \& SIEGeNTHAler 1976, Fritz et al. 1975, TAlbot 1990, EICHER et al. 1991, HoEFs 1996).

Die Paläoseen von Wilczyn und Ossówka sind detailliert palynologisch untersucht, wobei Pollendiagramme für sechs Kerne der fossilen Seesedimente aus Wilczyn (BINKA \& NitychoruK 1995, 1996, Krupinski 1995, Binka et al. 1997) sowie zwei Diagramme für Ossówka (KruPINSKI 1995) erarbeitet wurden. Das reiche palynologische Material erlaubt eine detaillierte Analyse möglicher Paläoklimaveränderungen und die Ableitung von Wasserspiegelschwankungen in dem Paläosee (Binka \& Nitychoruk 1996, Binka et al. 1997). Es ermöglicht ferner, bestimmte Abschnitte der Isotopenkurven konkreten Klimaschwankungen zuzuordnen. Die Isotopenkurven für den $55 \mathrm{~m}$ langen Kern in Ossówka sind in den Abbildungen 5 und 6 dargestellt. Die Phasen einer Abkühlung des Klimas sind mit Pfeilen (von 1 bis 6) auf der Abb. 5 vermerkt.

Die ältesten Proben von der Basis der Profile stammen aus einer Wechsellagerung von dunkelgrauen etwa $1 \mathrm{~cm}$ mächtigen Ton-Siltlagen mit hellgrauen Silt-Sandlagen; diese Ablagerung entstand unmittelbar nach dem Abschmelzen des Inlandeises der Elster-Vereisung. Aufgrund der palynologischen Befunde muß die Sedimentation der $2 \mathrm{~m}$ mächtigen Sedimentschicht in einem ziemlich trockenen und kühlen Klima der Tundra erfolgt sein. Die spärliche Flora erleichterte die Erosion und das Ausspülen von aus den Ufern des Paläosees stammendem Sedimentmaterial, das vorwiegend aus Geschiebelehm bestand (Abb. 3).

Die ziemlich hohen $218 \mathrm{O}-$ Werte (Abb. 5) zur Zeit des kalten subarktischen Klimas spiegeln daher 


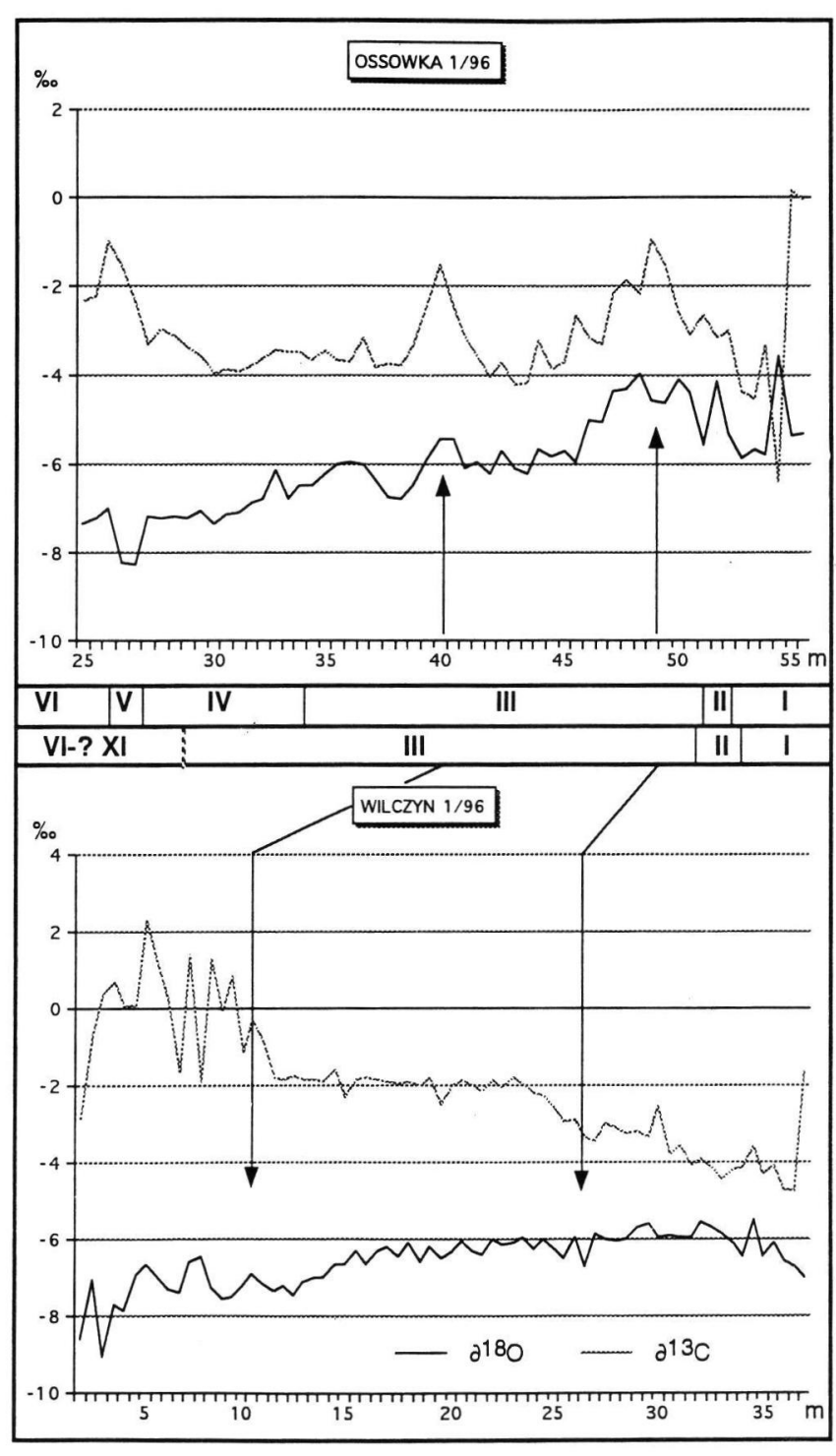

Abb. 6: Isotopen-Kurven des $13 \mathrm{C}$ und $18 \mathrm{O}$ für die Lokalitäten Ossówka und Wilczyn (Karbonat-Ablagerungen). Korrelation aufgrund des $\mathrm{CaCO}_{3}$-Gehaltes und der palynologischen Untersuchungen. I, II, III ... - Pollen-Perioden (nach KRUPINSKI 1995).

Fig. 6: The isotope curves of $13 \mathrm{C}$ and ${ }^{18} \mathrm{O}$ for Ossówka and Wilczyn sites (carbonate sediments). Correlation based on Ca$\mathrm{CO}_{3}$ and palynological analyses. I, II, III - pollen periods (KrUPINSKI 1995).

möglicherweise den klastischen Eintrag von paläozoischen bzw. mesozoischen marinen Karbonaten aus Moränenmaterial wider. Hohe $\partial 13 \mathrm{C}-$ Werte sprechen für geringe Aktivität des Phytoplanktons.
Die in dem Profil zu bemerkende plötzliche Sedimentationsänderung hin zu oliv-grauem Silt (sehr fein geschichtet, die dünnsten Schichten mit $0,5 \mathrm{~mm}$ Mächtigkeit; ca. $53,5 \mathrm{~m}$ Teufe) entspricht auch den plötzlichen Schwankungen der Isotopen-Kurve und dies sowohl für ${ }^{13} \mathrm{C}$ als auch für $18 \mathrm{O}$. Die $\partial 13 \mathrm{C}$ Werte erniedrigen sich von 0,0 bis $-6,0 \%$, und die $\partial 18 \mathrm{O}$-Werte steigen von $-6,2$ bis $-3,5 \%$ (Abb. 6). Diese Verschiebungen werden dahingehend gedeutet, daß aus einem flachen Becken ein eher tiefer See wurde. Die Änderung der hydrologischen Verhältnisse ging mit der Temperatursteigerung einher, die für den Beginn des Interglazials charakteristisch ist und mit dem Sprung NAP minus Pinus (in Kalilow, Woskrzenice und Komarno - Abb. 1) parallelisiert werden kann. Die Erwärmung führte zu einer intensiveren Verdunstung, was die $18 \mathrm{O}$ Anreicherung der Karbonate erklärt. Die Verschiebung der $\partial{ }^{13} \mathrm{C}$-Werte in Richtung auf eine $13 \mathrm{C}$-Verarmung kann durch Einfließen von Grund- und/oder Flußwasser bei einer insgesamt geringen Aktivität des Phytoplanktons verursacht sein (EICHER et al. 1991).

Eine ähnliche Erscheinung einer Zunahme der $\partial 18 \mathrm{O}$-Werte wird auch am Übergang vom späten Weichsel-Glazial zum Holozän in den Profilen aus dem Gosciaz-See (Kuc et al. 1993, Goslar et al. 1995) sowie in den Eiskernen aus Grönland (DANsGard et al. 1993) festgestellt.

Das Erscheinen und der Rückgang der Birken-Kiefern-Wälder (Zone A/B) geht mit einer Kontinentalisierung des Klimas einher (Abb. 5), d. h. mit höherer Trockenheit, und einer anschließenden Abkühlung. Dies führt nach einer kurzen, palynologisch nicht gut faßbaren Erwärmung ( $\partial 18 \mathrm{O}-3,5 \%$ bei $54,0 \mathrm{~m}) \mathrm{zu}$ einer nachfolgenden Verringerung der d 18O-Werte auf etwa -5,5\%0 (Abb. 4, 5), bedingt wahrscheinlich durch eine Abkühlung und eine Reduktion der Verdunstung. Das Verwischen einer deutlichen Schichtung in den Sedimenten zwischen $52,3 \mathrm{~m}$ und $53,5 \mathrm{~m}$ kann auf eine vorübergehende Verflachung des Palaeosees zurückgeführt werden (Abb. 2). Erhöhte Mengen an Schwefel in den Ablagerungen (Abb. 5) gehen mit der Sen- 